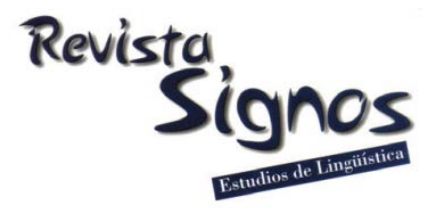

\title{
Habilidades metamorfológicas y su incidencia en la comprensión lectora
}

\author{
Metamorfological skills and their incidence on reading \\ comprehension
}

\author{
Yuri Vega Rodríguez Angélica María Torres \\ UNIVERSIDAD DEL Bío Bío \\ CHILE \\ yvega@ubiobio.cl
}

\author{
Manuel del Camp0 \\ Rivas \\ UNIVERSIDAD SANTO TOMÁS \\ CHILE \\ manuel.del.campo.12@gmail.com
}

Recibido: 22-VII-2015 / Aceptado: 09-XII-2016

\section{Resumen}

La conciencia morfológica se refiere al plano consciente de las estructuras morfémicas de las palabras y las habilidades para manejar y manipular dichas estructuras. Diferentes estudios, principalmente en habla inglesa, proponen que el procesamiento morfológico desempeña un rol importante en la adquisición de las habilidades lectoras, ya que es esencial para la comprensión del lenguaje. Por ello, el objetivo del presente estudio fue conocer si existe una relación de incidencia entre las habilidades metamorfológicas y la comprensión lectora. El estudio tuvo un enfoque cuantitativo, diseño observacional de tipo analítico correlacional y corte transversal. Se diseñó una Prueba de Evaluación de Habilidades Metamorfológicas (PEHMM), a la cual se le realizó validación de contenido con juicio de expertos y validación de criterio con el BLOC-S (Batería de Lenguaje Objetiva y Criterial - Screening), obteniéndose un instrumento con 4 subpruebas de análisis y composición morfológico; dicha prueba se aplicó a 36 estudiantes de 4to básico de educación regular, junto con la prueba CLP (Complejidad Lingüística Progresiva) para evaluar la comprensión lectora. Como hallazgo principal es posible afirmar que existe una relación entre las habilidades metamorfológicas y la comprensión lectora. Se ha evidenciado que las habilidades de análisis morfológico dentro de un contexto oracional presentan mayor relación con la lectura que la habilidad de derivar palabras a partir de una raíz. Al aplicarse regresión lineal, se demostró que existe una relación lineal entre los puntajes de la prueba 'CLP' y el rendimiento en la 'Prueba de Habilidades Metamorfológicas', el modelo explica alrededor el $42,32 \%$ de la variabilidad de los resultados en comprensión lectora.

Palabras Clave: Habilidades Metamorfológicas, Composición Morfológica, Análisis Morfológico, Comprensión de Lectura. 


\begin{abstract}
Morphological awareness is referred to the conscious level of the morphemic structure of words, and the skills to manage and manipulate these structures. Different studies, mainly in English, suggest that the morphological processing plays an important role in reading skills acquisition, essential for language comprehension. Therefore, the objective of the present research was to study if there exists a relation between metamorfological skills and reading comprehension, and to develop a predictive analysis model of reading comprehension from the metamorfological skills. This study had a quantitative approach, and was an analytical correlational and transversal study. A Metamorfological Skills Assessment Test (MSAT) was design incorporating content validation using expert judgment and criterion validation with BLOC-R, getting an instrument with 4 subtests of analysis and morphological composition. This instrument was applied to 36 4th grade primary school students, in conjunction with the CLP test (Progressive Linguistic Complexity) to assess reading comprehension. As part of the results, we found that there is a relation between metamorfological skills and reading comprehension. The results also revealed that the morphological analysis skills, within a sentence context, are far more related to reading than the skill of derived words from a single root. Lineal regression was applied and it showed that there is a lineal relation between the scores of the 'CLP' and performance in the 'Metamorfológicas Skills Assessment Test'. The model explains about $42.32 \%$ in variability of the results in reading comprehension.
\end{abstract}

Key Words: Metamorfological skills, morphological composition, morphological analysis, reading comprehension.

\title{
INTRODUCCIÓN
}

Las habilidades metamorfológicas entregan información sobre la composición de la forma de la palabra (a partir de la conciencia de su estructura) y permiten realizar inferencias de significado; constituyen por lo tanto, habilidades esenciales en ámbitos significativos del aprendizaje como la comprensión lectora (Carlisle, 2003; Stone, Silliman, Ehren \& Apel, 2004; Díaz, 2006; Duncan, Casalis \& Colé, 2008). En efecto, las habilidades metamorfológicas (de análisis y composición de la palabra), hacen posible el uso consciente de procesos cognitivos que facilitan la comprensión lectora.

Al analizar el currículum escolar, se espera que los estudiantes de cuarto año de Educación Básica en Chile, desplieguen estrategias de comprensión que les permita "enfrentarse a la lectura de manera metacognitiva y reflexionar sobre las dificultades que surgen al leer" (MINEDUC, 2013: 38), ello invita a los docentes a adquirir conocimientos asociados a procesos de aprendizaje, con objeto de favorecer la implementación de estrategias efectivas en el aula.

La Organización para el Desarrollo y Cooperación Económica en su documento Literacy in the Information Age (2000), informa que en cuanto al nivel de comprensión lectora, el 80\% de los chilenos entre 16 y 65 años no posee el nivel mínimo de habilidad lectora, para funcionar en la sociedad actual, y que solo el 2\% alcanza un nivel aceptable para vivir y desarrollarse en el mundo de hoy (OCDE Statistics 
Canadá, 2000), revelando la urgencia de estudios y metodologías que permitan revertir esta situación.

La comprensión de lectura es una habilidad compleja en la que intervienen múltiples factores, dentro de los cuales las habilidades lingüísticas y cognitivas ostentan un papel fundamental. En esta línea, la literatura (principalmente inglesa) indica que en parte, el éxito de un individuo en comprensión lectora, se debe a las habilidades metamorfológicas que posee (Anglin, 1993; Carlisle, 1995); sin embargo, las referencias en este punto son escasas para el habla hispana.

Considerando los antecedentes anteriormente expuestos, los objetivos de este estudio son: conocer si existe una relación de incidencia entre las habilidades metamorfológicas y la comprensión lectora, adicionalmente crear un modelo predictivo de la comprensión lectora a partir de las variables antes mencionadas.

\section{Marco teórico}

Para leer y escribir se realizan una serie de operaciones cognitivas que provienen de niveles previos del desarrollo del lenguaje (de tipo oral específicamente). Para establecer la relación entre los sistemas, el individuo recurre a la Conciencia Lingüística, o Habilidad Metalingüística, que se podría definir como el conocimiento que posee acerca del lenguaje en cada uno de sus niveles (pragmático, semántico, sintáctico, morfológico y fonológico), permitiéndole conocer y manipular cognitivamente el lenguaje y la actividad lingüística de un interlocutor y de sí mismo. Aun cuando cada uno de estos niveles lingüísticos se encuentra en directa relación con el aprendizaje lector, es la conciencia metamorfológica la que permite comprender la composición estructural de palabras, permitiendo manipular su estructura y reflexionar sobre su significado, otorgando herramientas que favorecen la comprensión general durante la lectura. La fonología y la ortografía explican solo una parte de la capacidad para leer y deletrear, primordialmente en las etapas iniciales de adquisición de dichas habilidades, es en particular la conciencia morfológica la que tiene un papel relevante dentro de las variables que explican el éxito en la comprensión lectora en niveles más avanzados de escolaridad (Díaz, 2006).

\subsection{Comprensión lectora}

Existen diversos modelos que tratan de explicar el proceso lector (González, Barba \& González, 2010), donde la lectura se redefine, en términos de reconocimiento y comprensión como un proceso constructivo e inferencial que consolida y verifica hipótesis acerca de determinados signos, gráficos, letras y palabras, descifrando su significado a través de la representación mental y la asignación de un valor lingüístico a cada uno de éstos dentro de un contexto. 
Desde la perspectiva cognitiva la comprensión lectora se define como un proceso y un producto, a través del cual, el lector elabora un significado en su interacción con el texto (Anderson \& Pearson, 1984) requiriendo la aplicación específica de destrezas y estrategias cognitivas de carácter general, lo que equivaldría a la creación, modificación, elaboración e integración de las estructuras de conocimiento, almacenándolas en la memoria a largo plazo (Pérez, 2005). Es gracias a la memoria a largo plazo que se establecen vínculos de significados con otros conocimientos previamente adquiridos en el momento de la lectura, con lo cual se van construyendo aprendizajes significativos sobre los esquemas cognitivos (Ausubel, Novack \& Hanesian, 1983). Otro tipo de memoria vinculada en el proceso de comprensión lectora es la memoria a corto plazo. Al leer se activa el mecanismo de asociación, secuenciación, linealidad y recuerdo del texto, siguiendo la trayectoria o disposición lógica de la lectura a medida que se va leyendo, con ello se produce un proceso continuo de memoria inmediata al ir evocando los nuevos contenidos, acciones o escenas textuales que aparecen con los respectivos personajes, temas, acciones u otros datos expresados en el texto. Así los dos tipos de memoria, producen interconexiones significativas entre las diferentes partes del texto leído, contribuyendo a una buena comprensión de lo que se lee (Cuetos \& Valle, 1988). No obstante, es en la memoria a largo plazo donde el lector recobra la información semántica y sintáctica disponible, a través del acceso al léxico que se lleva a cabo por medio diferentes rutas (Vallés, 2005). Es en este acceso al almacén léxico donde recopilamos la información acerca de las estructuras de tipo pragmático, semántico y morfológico que dan sentido a la lectura, prevaleciendo unas sobre otras dependiendo del objetivo del lector (Vallés, 2005).

Después de acceder al léxico, se da paso al análisis de las relaciones estructurales entre las palabras que constituyen las frases, obteniendo la información contenida en el mensaje escrito. Este análisis sintáctico se realiza a través de la identificación de señales lingüísticas tales como: orden de la frase, estructura de la palabra y su función sintáctica, prefijos, sufijos y la conjunción de todas las señales lingüísticas orientadas a una sola interpretación o unidad global (Escudero, 2010), luego se realiza un análisis de las marcas lingüísticas a través del acceso sintáctico inmediato de cada palabra, y por último la memoria de trabajo condiciona la integración del sentido del texto que se lee (almacén de interpretaciones sintácticas ambiguas según González, 2004), asumiendo que mientras mayor sea la capacidad de ésta, mayor será la comprensión lectora (Cuetos \& Valle, 1988).

Luego de pasar por estas dos fases, el acceso al léxico y el análisis sintáctico, debe producirse la representación de la información expresada en el texto, es decir la comprensión lectora, la cual se consigue mediante una representación abstracta, formada por las unidades proposicionales, los personajes y sus roles y las circunstancias como el tiempo y el espacio (Escudero, 2010). Es en esta etapa donde el lector realiza las inferencias, definidas como procesos cognitivos mediante los cuales 
se obtiene información nueva del texto, basándose en la interpretación de la lectura de acuerdo con el contexto.

Este proceso nos permite afirmar que la comprensión de textos representa un comportamiento complejo, que implica el uso tanto consciente como inconsciente de diversas habilidades y estrategias a través de las cuales:

"los lectores monitorizan o auto-controlan sus propios progresos en el proceso de comprensión del texto, detectan los fallos que cometen y utilizan procedimientos que permiten la rectificación de dichos fallos" (Pérez, 2005: 122).

\subsection{Habilidades metalingüísticas}

La metalingüística se desarrolla en forma natural como consecuencia del aprendizaje del lenguaje. Diferentes estudios sugieren que la conciencia del lenguaje se desarrolla en fases o estados, los cuales podrían tener conexión con el desarrollo cognitivo y el acceso a experiencias facilitadoras como la enseñanza de la lectura (Duncan et al., 2008).

Son tres las fases identificadas en el desarrollo de la conciencia del lenguaje (Valtín, 1984): la primera de ellas es la conciencia inconsciente o uso automático del lenguaje; la segunda la conciencia real, que implica la idea de que el niño poco a poco es capaz de pensar en las prioridades del lenguaje, pero el conocimiento de las unidades del mismo aún es implícito; y por último la conciencia consciente, valga la redundancia, la cual lleva a creer que los niños podrían manipular deliberadamente las unidades del lenguaje a través de las habilidades metalingüísticas (García \& González, 2008).

Muchos autores han reconocido la implicancia que poseen las Habilidades metalingüísticas en el proceso lector, y más aún, en la comprensión de lectura, pues éstas representan la destreza por parte del lector, no solo de conocer la estructura del texto, sino de ser consciente de amplios procesos lingüísticos que se desencadenan al momento de leer (Florez, Torrado, Arévalo, Mesa, Mondragón \& Pérez, 2005).

Dentro de éstas habilidades se pueden encontrar tres componentes: (1) el conocimiento declarativo o conocimiento 'de hecho', sobre los aspectos lingüísticos del texto que se lee; (2) el conocimiento procedimental de 'cómo usar' el conocimiento declarativo, y (3) el conocimiento condicional sobre 'cuándo utilizar' el conocimiento declarativo, qué procedimiento usar y por qué es importante (Paris, Cross \& Lipson, 1984; Schunk, 2005).

Las habilidades de conciencia morfológica, en relación a las habilidades metalingüísticas, han sido fuertemente relacionadas con el éxito de la comprensión lectora en diferentes países de habla inglesa (Stone et al., 2004; Carlisle, 2006; Díaz, 
2006, Duncan et al., 2008). Debido a su relevancia, se realizará a continuación una revisión sobre éstas.

\subsection{Habilidades metamorfológicas de análisis y de composición morfológica}

En la edad escolar la conciencia morfológica, en el aprendizaje de la utilización de una regla morfológica, tiene su origen en la hipótesis de que algunas palabras deben ser tratadas gramaticalmente de cierta manera. El primer uso de un señalizador morfológico tiene su base en alguna forma de memorización mecánica, de la cual se realizará una generalización y por ende se finalizará con el establecimiento de la regla (Bybee \& Slobin, 1982). El desarrollo de la Conciencia Morfológica se manifiesta inicialmente de forma implícita, como ocurre con los niños de edad preescolar en el lenguaje oral (Berko, 1958), y después de forma explícita cuando el niño es capaz de analizar las estructuras morfológicas de las palabras aisladas, apareciendo los primeros signos de conciencia morfológica en la escuela primaria (Nicasio \& González, 2006). Basándose en revisiones de estudios más extensos, se infiere que la morfología flexiva se alcanza tempranamente en el desarrollo evolutivo y es el tipo de morfología más frecuentemente examinada en habilidades lectoras en niños de $1^{\circ}$ y $2^{\circ}$ básico. Los morfemas flexivos o gramaticales, no modifican el significado de la raíz sino que aportan nociones gramaticales (género, número, tiempo) relacionando una raíz con otras, (1) ejemplo: 'las niñas bailan'. En el primer grado, la mayoría de los niños utilizan adecuadamente las reglas morfo-fonológicas para la formación de las inflexiones, como se muestra en el estudio clásico de Berko (1958) sobre la producción oral de los niños.

Por otro lado, la morfología derivativa implica un periodo más largo pero con un final más abierto y con mayor variabilidad, este hecho explica la asociación consistentemente mostrada entre el conocimiento de morfemas derivativos y habilidades lectoras en cursos superiores (Mahony, Singson \& Mann, 2000). Los morfemas derivativos o afijos modifican y concretan el significado de la raíz. Según su posición pueden ser prefijos (los que se ubican delante de la raíz), sufijos (se ubican detrás de la raíz), o interfijos (los que se ubican entre la raíz y un sufijo).

Desde la década de los cincuenta se ha observado que los niños en edad preescolar y primer grado de estudio no muestran un conocimiento productivo de los principios de derivación ante el requerimiento de formar palabras compuestas en tareas particulares (Berko, 1958). En las derivaciones, más que en las inflexiones, la complejidad de las relaciones morfo-fonológicas, hace que sea difícil para los niños apreciar la estructura morfológica de muchas palabras (Carlisle, 2006).

La habilidad de composición de la morfología léxica junto a la derivación se insertan en el segundo lapso temporal de la evolución más tardía (Owens, 2003). Así, en $3^{\circ}$ y $5^{\circ}$ año básico escolar, es muy importante la consolidación de la morfología 
derivativa a través de los sufijos, pero es más esencial aún el desarrollo de la adquisición de los principales prefijos de inflexión (González, Rodríguez \& Gázquez, 2011).

En las habilidades de conciencia morfológica existen dos variables que juegan un papel importante en la forma en que los niños infieren el estado morfológico de una palabra: el análisis morfológico y la composición morfológica (Stone et al., 2004). Las habilidades metalingüísticas de composición morfológica, a partir de lo planteado por Stone et al. (2004), se entienden como las habilidades correspondientes a la capacidad consciente del niño para derivar palabras a partir de otras. Las habilidades de análisis morfológico por su parte, son las habilidades de conciencia morfológica que implican el análisis para determinar la palabra de la cual se ha derivado otra.

\subsection{Habilidades metamorfológicas y el currículo nacional}

Para monitorear la adquisición de los aprendizajes, en Chile se determinan a partir de los programas de estudio generados por el Ministerio de Educación, los objetivos de aprendizaje de cada una de las asignaturas del marco curricular nacional, así como las orientaciones para planificar y evaluar dichos aprendizajes (MINEDUC, 2012).

Respecto a la adquisición de las habilidades metamorfológicas en el currículo nacional, se mencionan dentro de los objetivos y aprendizajes de primero y segundo básico el dominio de formas que representen el plural, también se hace alusión a la morfología flexiva, a partir del uso de aumentativos y diminutivos, vinculados con los textos utilizados en tareas de comprensión lectora. El aprendizaje lingüístico esperado en tercer y cuarto año básico menciona el uso de vocabulario frecuente y de palabras no familiares, en las que se hace imprescindible el manejo de prefijos y sufijos que faciliten su comprensión.

En este periodo se inicia la adquisición de la morfología derivativa, compuesta por sufijos de derivación, formación de palabras compuestas y prefijos de inflexión, a partir de un aumento considerable de la presentación de este tipo de estímulos en el aula y en los textos escolares. Este aumento constante de la exposición de los niños en edad escolar a la adquisición de palabras morfológicamente complejas a través de su aprendizaje (Anglin, 1993), puede explicar por qué la conciencia morfológica jugaría un papel progresivamente más importante en el desarrollo del vocabulario y en la lectura de palabras.

\section{Marco metodológico}

El tipo y diseño del presente estudio es descriptivo correlacional, no experimental de corte transversal (Hernández, Fernández \& Baptista, 2010). 


\subsection{Hipótesis del estudio}

H1: Existe relación de incidencia de las Habilidades Metamorfológicas en la comprensión lectora, en estudiantes de 4 año básico.

H0: No existe relación de incidencia de las Habilidades Metamorfológicas en la comprensión lectora, en estudiantes de 4 año básico.

\subsection{Participantes y criterios de selección}

Se incluyó a: a) estudiantes regulares que no asistían a proyectos de integración por educación especial; b) estudiantes sin diagnósticos de problemas de aprendizaje debido a dificultades lingüísticas, cognitivas, neurológicas, sensoriales, y/o motrices, verificados con los informes, psicológicos y psicopedagógicos de cada uno de los niños aportados por las instituciones, c) estudiantes sin antecedentes de repitencia escolar, d) estudiantes cuyo consentimiento informado fue firmado por los apoderados, e) estudiantes que hayan rendido ambas pruebas del estudio.

Tras aplicar los criterios de inclusión a una matrícula de 63 estudiantes de cuarto año básico, de los establecimientos educacionales regulares urbanos: Arturo Pacheco Altamirano y Tomás Lago (dependientes del Departamento Administrativo de Educación Municipal de Chillan Viejo, región del Bío Bío); se obtuvo una población de 36 menores, 19 niños y 17 niñas.

El levantamiento de los datos fue autorizado por la Directora del Departamento Administrativo de Educación Municipal (DAEM), por los directores de los establecimientos y por los padres de los niños, quienes debieron firmar un consentimiento informado que apoyó la participación de los menores en el estudio.

\subsection{Instrumentos}

Para medir la comprensión lectora, se usó la Prueba de Comprensión Lectora de Complejidad Lingüística Progresiva (Alliende, Condemarín \& Milicic, 1991) (CLP), y para evaluar las habilidades metamorfológicas se diseñó la Prueba de Evaluación de Habilidades Metamorfológicas (PEHMM).

\subsubsection{Diseño del instrumento}

Prueba de Evaluación de Habilidades Metamorfológicas (PEHMM), instrumento inédito diseñado para el presente estudio, y generado en tres fases: La primera consideró una revisión de los objetivos planteados por los Planes y Programas del Ministerio de Educación para el cuarto año de educación básica (MINEDUC, 2007) y el análisis de las Habilidades Metamorfológicas implícitas en ellos. La segunda fase consistió en construir una base de datos con las palabras que utilizan frecuentemente los menores, obtenidas de la Lista de Frecuencias de Palabras del Castellano de Chile (LIFCACH) desarrollado por Sadowsky y Martinez (2004), además, se extrajeron 
palabras de los libros de Lenguaje, Matemáticas, Historia, Comprensión del medio y sociedad de $3^{\circ}$ y $4^{\circ}$ año del Gobierno de Chile, posteriormente se seleccionaron las palabras que coincidían en ambos listados. La tercera fase consistió en construir tareas y clasificarlas en subpruebas del instrumento. Las subpruebas se construyeron teniendo en cuenta las habilidades de conciencia morfológica, en sus dimensiones de análisis y composición. El resultado fueron 7 subpruebas con 30 reactivos cada una, para un total de 210 ítems a validar. Cada subprueba además contaba con dos ejemplos para explicar la instrucción de la tarea.

\subsubsection{Métodos de validación de la Prueba}

- Validez de contenido: Una vez confeccionada la estructura general del instrumento, se realizó validación de contenido usando el método de Lawshe (1975). Se seleccionaron a 8 expertos, de profesión fonoaudiólogos quienes hubieran trabajado previamente con estudiantes entre 8 y 12 años en el área de lenguaje infantil. A cada juez experto se le entregó una copia de la prueba junto a un manual que contenía las instrucciones para cada subprueba, además de la explicación teórica y gráfica de las partes constitutivas del instrumento en cuestión. Cada juez experto en consideración de los criterios teóricos propuestos, valoró cada reactivo como Esencial (E), útil pero No esencial (UNE) y No necesario (NN). Se validaron aquellos reactivos que obtuvieron un valor igual o superior a $75 \%$ de aceptación, quedando como resultado una Prueba con 4 subpruebas: cada una de ellas con 15 reactivos, organizados de la siguiente forma:

Habilidad metamorfológica de composición morfológica:

- Subprueba $\mathrm{N}^{\mathrm{o}}$ 1: Construye palabras usando morfemas derivativos a partir de una palabra aislada.

- Subprueba $\mathrm{N}^{\mathrm{o}}$ 2: Deriva palabras situadas en un contexto oracional.

- Subprueba $\mathrm{N}^{\circ}$ 3: Construye pseudopalabras derivadas a partir una pseudopalabra.

Habilidad metamorfológica de análisis morfológico:

- Subprueba $\mathrm{N}^{0}$ 4: Identifica la palabra de la que se deriva el estímulo que se le presenta.

- Validez de criterio (concurrente): con el objetivo de determinar la validez de criterio, se pidió a los 36 participantes responder la prueba de habilidades metamorfológicas (PEHMM) y como gold estándar, la sub prueba de Morfología de la Batería de Lenguaje Objetiva y Criterial- Screening: BLOC-S (Puyuelo, 2007). 
Estas pruebas fueron administradas a los menores en la biblioteca del colegio, un lugar con buena iluminación y sin ruido; se aplicaron de forma individual, en 1:1. Se consideraron momentos diferentes para realizar cada prueba y así evitar la fatiga por parte de los estudiantes, en un tiempo no mayor de 3 semanas (una prueba cada semana). Los datos fueron recopilados en conjunto con estudiantes de pregrado de la carrera de Fonoaudiología de la Universidad del Bío-Bío que realizaban una tesis en la misma línea investigativa y que aportó información al presente estudio. Una vez obtenidas las respuestas de ambos instrumentos, se calculó el coeficiente de correlación de Pearson entre los dos instrumentos, con el objetivo de evaluar el grado de concordancia de ambas pruebas.

Para correlacionar los resultados de las pruebas CLP y PEHMM obtenidos por los estudiantes, se utilizó el coeficiente de correlación de Pearson. La normalidad de los datos fue comprobada con la prueba de Shapiro-Wilk, se utilizó un nivel de significación de 0,05. El procesamiento de los datos fue gestionado con Stata versión 11.0 .

\section{Resultados}

Las variables 'Comprensión Lectora', medida a través de la CLP y 'Habilidades Metamorfológicas', medida con PHMM, presentaron datos con distribución normal $(\mathrm{Z}=1.608, \mathrm{P}=0.054$ y $\mathrm{Z}=1.395, \mathrm{P}=0.0815$, respectivamente $)$

El Gráfico 1 muestra una tendencia directamente proporcional entre la comprensión lectora (prueba CLP) y las habilidades metamorfológicas (PHMM), con un $\mathrm{r}=0.66(\mathrm{p}<0.01)$.

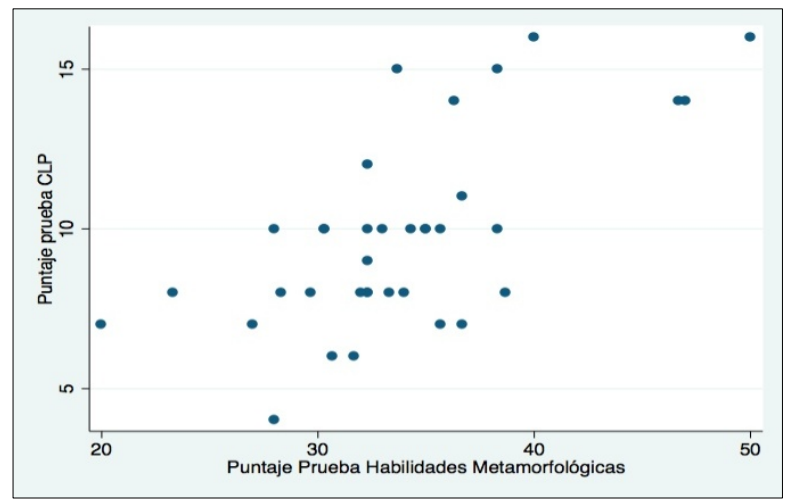

Gráfico 1. Puntaje en la Prueba de Habilidades Metamorfológicas versus resultados de la prueba CLP.

Al correlacionar la comprensión lectora (CLP) con cada una de las subpruebas de la PEHMM (Gráfico 2), se observó que entre la CLP y la subprueba №1 
(construcción de palabras a partir de una palabra raíz), se presentó un $\mathrm{r}=0.33$ ( $\mathrm{p}>0.05$ ). Respecto a la correlación del CLP y la subprueba No2 (completación de oraciones con una palabra derivada), de la PHMM, se obtuvo un $r=0.53(p<0.01)$. Para la subprueba $\mathrm{N}^{\circ} 3$ de la PHMM (encontrar la raíz de una palabra), se obtuvo un $\mathrm{r}=0.52(\mathrm{p}<0.01)$. Finalmente, entre la CLP y la subprueba $\mathrm{N}^{\circ} 4$ (construcción de palabras derivadas a partir de una pseudo palabra), obtuvo un $\mathrm{r}=0.50(\mathrm{p}<0.05)$.

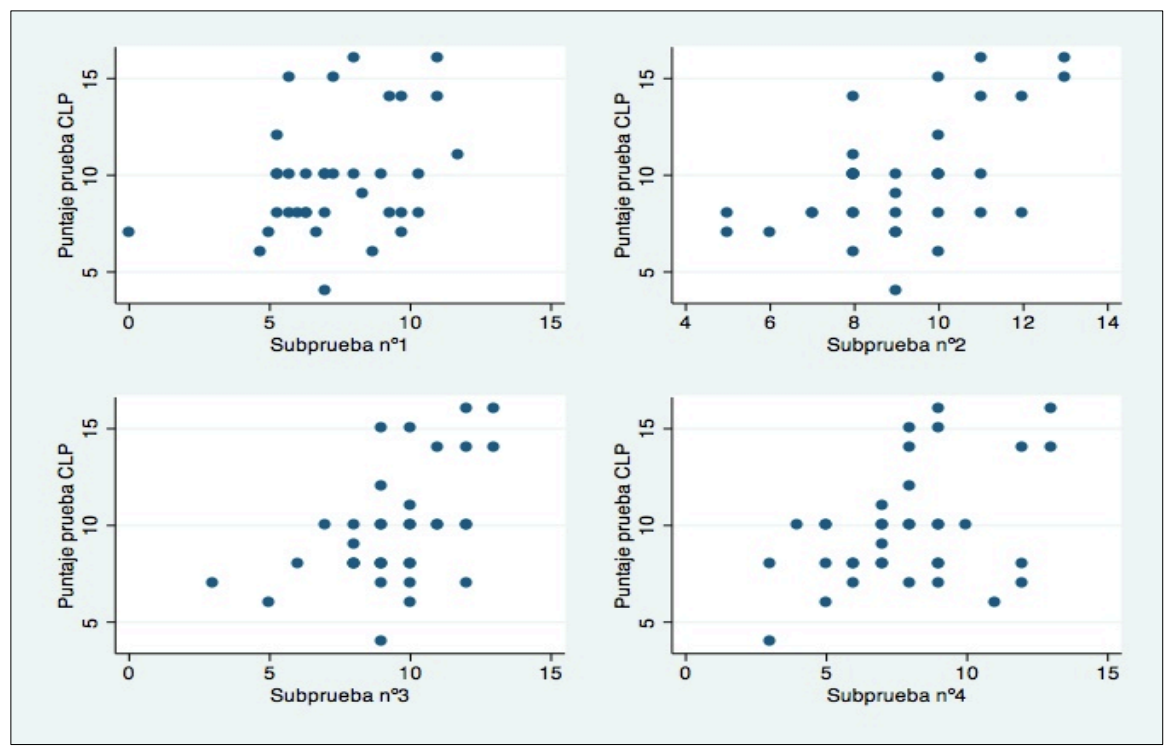

Grafico 2. Rendimiento en la prueba CLP versus el obtenido en las subpruebas de la Prueba de Evaluación de Habilidades Metamorfológicas.

El Gráfico 3 muestra una relación lineal entre los puntajes de la prueba CLP y el rendimiento en la PEHMM, $(\mathrm{F}=26.68, \mathrm{p}<0.001)$. De acuerdo al modelo lineal, la PEHMM logra explicar el $42,32 \%$ de la varianza de CLP, siendo estadísticamente significativo $(\mathrm{t}=5.17, \mathrm{p}<0.001)$.

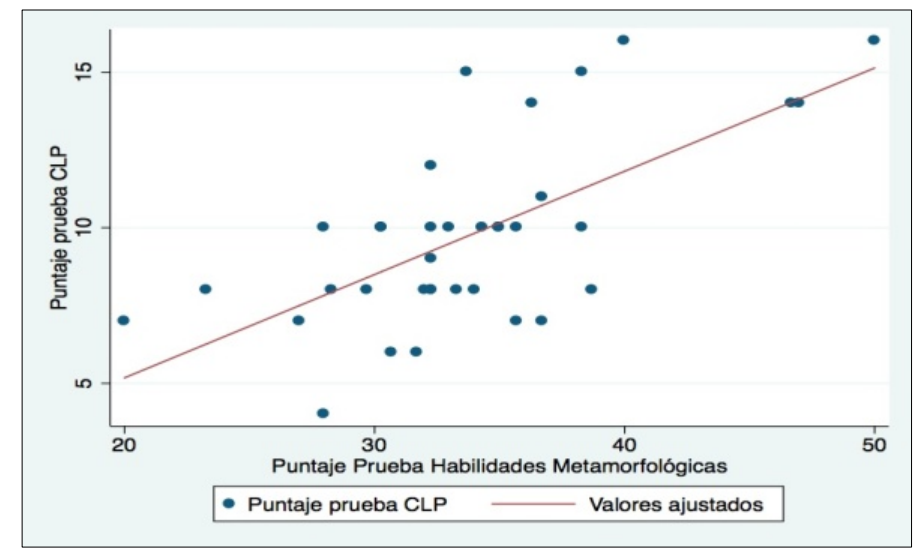


Grafico 3. Gráfico de dispersión de ambas variables con curva ajustada de valores ajustados.

\section{Discusión}

A partir de los datos obtenidos, es posible afirmar que existe una correlación entre las habilidades metamorfológicas y la comprensión lectora, de tal manera que cuanto mejor es el desempeño en las habilidades metamorfológicas mejor es el desempeño en tareas de comprensión lectora. De esta manera se aporta evidencia que relaciona el éxito de la comprensión de textos en hablantes del español de Chile con este tipo de habilidades lingüísticas, coincidiendo con lo mencionado por Anglin (1993), Nagy, Anderson, Schommer, Scott y Stallman (1989) y Carlisle (1995), entre otros, en investigaciones similares en idiomas extranjeros. Con los análisis de datos se pudo establecer que al incremento en un punto de la Prueba de Habilidades Metamorfológicas se espera un aumento de 0.33 puntos en la prueba CLP.

De acuerdo a la regresión lineal entre los puntajes de la prueba CLP y el rendimiento en la PEHMM $(\mathrm{F}=26.68, \mathrm{p}<0.001)$, los resultados indican que las habilidades metamorfológicas explican en gran parte el éxito en la comprensión lectora, sin embargo, entendiendo la complejidad de dicha tarea y los múltiples recursos cognitivos que involucra, es necesario estudiar otras variables que generen un modelo explicativo más amplio. Al analizar las correlaciones entre desempeño en la comprensión lectora y cada una de las tareas que se presentan en la PEHMM, se puede afirmar que la habilidad que los niños ponen en marcha en la construcción de palabras a partir de una raíz (habilidad de composición morfológica presentada en la primera subprueba), evidencia una relación débil con el desempeño en el proceso de comprensión de lectura, rechazándose la hipótesis que afirmaba la existencia de una relación de incidencia de la Habilidad Metamorfológica de composición morfológica en palabras aisladas en la comprensión lectora. Esto indica que la tarea de evocar palabras realizando derivaciones a partir de una palabra inicial, exige el uso de habilidades morfológicas de composición de palabras, pero no una conciencia propia del significado de las mismas, lo que resultaría en habilidades que no interfieren o no se encuentran relacionadas con la comprensión como tal. Lo anterior verifica la importancia de considerar el aprendizaje en contextos significativos que proporcionen a los estudiantes mayores oportunidades de construcción de significado de las palabras y su vinculación con otras palabras, elaborando redes semánticas más complejas

En cuanto a la segunda tarea: completar una oración con una palabra derivada, se encontró una relación más fuerte y estadísticamente significativa. El resultado permite aceptar la hipótesis que menciona que existe relación de incidencia de la Habilidad Metamorfológica de composición morfológica en palabras dentro de un contexto oracional con la compresión lectora. Este tipo de habilidades le permitirían al niño fácilmente entender significados, a partir de la forma de las palabras, situados en un 
contexto significativo, lo que claramente contribuiría a la comprensión de textos. En contraste con la anterior tarea, el analizar las palabras dentro de un contexto, valiéndose de la información que rodea la palabra, en este caso la oración, permite al niño hacer un uso consciente de la derivación, relacionando la forma de la palabra con el contenido de las mismas. De esta manera, en el momento en que el niño que se ve enfrentado en un texto a una palabra desconocida, logrará analizar su forma y comprender su significado, enriqueciendo su contenido gracias a la contribución que brinda la información adyacente.

A partir de esta información, es posible mencionar que la descontextualización de la enseñanza de las nociones léxicas, morfológicas y sintácticas, la falta de significatividad en su aprendizaje y el convertirse en un contenido más del currículo, no permiten que los estudiantes hagan un uso consciente de estas habilidades y que así las conviertan en herramientas que beneficien su aprendizaje. No es suficiente el conocimiento de los aspectos discursivos, lingüísticos y textuales, sino que también es preciso dominar en contextos significativos los procesos implicados en la comprensión.

Con respecto a la tercera tarea: identificación de la raíz morfológica de una palabra, en la que el niño debe usar la habilidad de análisis metamorfológico, se encontró una correlación altamente significativa con la comprensión lectora, por lo que se acepta la hipótesis que afirma la existencia de una relación de incidencia de la Habilidad Metamorfológica de análisis morfológico en la comprensión lectora, evidenciado que el proceso que requiere el niño para descomponer y analizar el origen o la raíz de una palabra que se le presenta, le permitirá en tareas de comprensión lectora, a partir de la información morfológica de la palabra, superar obstáculos que puedan disminuir o limitar la apropiación de un texto, como el enfrentarse a palabras de las que no conoce el significado.

A partir de lo anterior, es necesario que el niño conozca que las palabras están formadas por unidades más pequeñas de significado y que le dan sentido a éstas. En esta actividad es relevante que los niños tengan conciencia de que las palabras tienen raíces, pues independiente del cambio de significado que pueda otorgar el sufijo, hay un componente semántico que se mantiene y que inicialmente permite tener un referente léxico de la palabra, para luego, a partir de la conciencia de su derivación, deducir su significado. Es importante mencionar que la comprensión de la derivación por sí sola no podría garantizar el éxito en la extracción del significado de una palabra, pues si no se conoce su origen, solo se comprenderá parte del mismo.

La metamorfología como proceso, debe ser apoyada por habilidades metasemánticas y metasintácticas, entre otras, con objeto de garantizar la lectura y su comprensión (Armbruster, Lehr \& Osborn, 2001; Kamhi \& Catts, 2005) ya que el 
complemento que brindan cada una de estas capacidades en la tarea de la interpretación, resulta esencial en la tarea de comprensión.

$\mathrm{Al}$ analizar la cuarta y última tarea en la que se despeja la carga semántica y se busca la composición metamorfológica pura a partir del uso de no palabras, se acepta la hipótesis que relaciona la habilidad metamorfológica de composición morfológica de pseudopalabras con la compresión lectora. Este resultado pone de manifiesto una vez más que las habilidades de análisis morfológico de la palabra, aislando las posibles dificultades que pueden devenir de la semántica (como el desconocer el significado), le permiten al niño hacer deducciones de contenido, entendiendo que aunque la palabra no exista o no sea conocida para un sujeto, se pueden designar significados a partir de las derivaciones. Por ejemplo, para nombrar a una persona que realiza un oficio, sabemos que debemos adicionar alguno de los morfemas derivativos 'ero', 'ista' u 'or', entendiendo que las opciones en nuestra lengua son finitas; de esta manera prevalecen habilidades de conciencia de la forma, más que habilidades particulares que puedan devenir del léxico o vocabulario. Tareas que impliquen este tipo de análisis favorecen la comprensión lectora, pues le brindan al niño la posibilidad de deducir significados y por lo tanto no se hace necesario conocer todas las palabras que aparecen en un texto. Adicionalmente, se disminuye el problema que conlleva enfrentarse a palabras nuevas, puesto que el análisis del significado a partir de la forma, permitiría que la comprensión del texto no se viese mayormente afectada.

Esta tarea pone de manifiesto la importancia del conocimiento de sufijos y prefijos, pues permite que el niño comprenda que al cambiar la parte final de una palabra puede generar cambios en su significado, de esta manera podríamos afirmar que conocer explícitamente las derivaciones y los significados de éstas, contribuye positivamente en la comprensión de un texto.

A partir de los resultados de la presente investigación se puede reafirmar que en el habla hispana al igual que en la lengua inglesa, las habilidades de conciencia morfológica tienen un papel importante en la comprensión de textos. Casalis y LouisAlexandre (2000), indican que el desarrollo tanto de la lectura y su comprensión como de la conciencia morfológica, no son independientes de la forma que los análisis morfológicos en educación infantil predicen el nivel de lectura en los cursos de primero y segundo de primaria, contribuyendo según Deacon y Kirby (2004), de manera sutil pero significativa, en la comprensión lectora de la lengua española. Si se considera que los escolares se enfrentan durante su aprendizaje a palabras que son morfológicamente complejas (Anglin, 1993; Nagy et al., 1989) y que esto explica por qué la conciencia morfológica juega un papel progresivo e importante en el desarrollo del vocabulario (Nagy \& Scott, 2000; Carlisle, 2007), investigaciones, como las presentadas en este trabajo, evidencian la necesidad de generar estrategias didácticas que refuercen este tipo de habilidades lingüísticas y garanticen un mejor acercamiento a los textos. 
De acuerdo a lo mencionado por Bowey y Patel (1988), se puede ratificar que la correlación significativa entre ambos factores (lectura, su comprensión y habilidades metamorfológicas) podría tratarse de un reflejo y no tanto de un conocimiento de los componentes del lenguaje, sino más bien una actitud general que va más allá de las formas del lenguaje y su uso. Esta actitud general implica el desarrollo continuo de la experiencia cognitiva, que según Carretero (2001), se relaciona con procesos de supervisión y de regulación ejercidos sobre su propia actividad cognitiva, cuando se enfrentan a tareas tan complejas como la comprensión lectora, necesitando la supervisión de diferentes metaprocesos, como en este caso, la habilidad metamorfológica.

\section{CONCLUSIONES}

De acuerdo a lo desarrollado en este trabajo, las habilidades metamorfológicas se convierten en un punto de investigación relevante, desde el cual se intenta entender la manera en que los niños y niñas adquieren conciencia de cambios de significados a partir de las marcas morfológicas de las palabras (morfemas, sufijos, prefijos). Los niños escolares entre 6 y 11 años se ven enfrentados a la definición de palabras morfológicamente complejas y esta situación afecta la adecuada comprensión de lectura (Carlisle, 1995). Dicha información es posible corroborarla al analizar los textos otorgados por el gobierno de Chile correspondientes a los niveles de cuarto y quinto año básico en áreas de lenguaje, matemáticas y comprensión del medio, en los que se presentan palabras nuevas de gran complejidad morfológica, en las que el acceso a su significado y comprensión podrían mejorar en la medida que se vincularan de forma más explícita la enseñanza de habilidades que impliquen el uso consciente de recursos cognitivos y lingüísticos y metalingüísticos. A pesar del uso correcto que pueden hacer los niños de diferentes habilidades lingüísticas, no implica que sean conscientes de ellas, ni mucho menos que las puedan utilizar como una herramienta que les permita apropiarse de otros contenidos y que beneficien el desempeño en otro tipo de competencias, como es el caso de la comprensión lectora. Es posible mencionar que la descontextualización de la enseñanza de las nociones léxicas, morfológicas y sintácticas, la falta de significatividad en su aprendizaje y el constituir un contenido más del currículo sin reflexión sobre el mismo, no permite que los estudiantes hagan un uso consciente de estas nociones, y que se beneficie el aprendizaje en general. No es suficiente el conocimiento de los aspectos discursivos, lingüísticos y textuales, sino que también es preciso dominar en contextos significativos los procesos implicados en la comprensión. Es por ello que emerge la importancia de una evaluación efectiva y la creación de estrategias tendientes a mejorar las habilidades que subyacen a los procesos complejos de aprendizaje. 


\section{REFERENCIAS BIBLIOGRÁFICAS}

Alliende, F., Condemartín, M. \& Milicic, N. (1991). Prueba CLP formas paralelas: Prueba de Comprensión Lectora de Complejidad Lingüística Progresiva: 8 niveles de lectura. Santiago: Ediciones UC.

Anglin, J. (1993). Vocabulary development: A morphological analysis. Monographs of the Society of Research in Child Development, 58(10), 238.

Anderson, R. \& Pearson, P. (1984). A schema-theoretic view of basic processes in reading comprehension. En P. Pearson (Ed.), Handbook ofreading research (pp. 255-291). Nueva York: Longman.

Armbruster, B., Lehr, F. \& Osborn, J. (2001). Put reading first: The research building blocks for teaching children to read. Washington, DC: National Institute for Literacy.

Ausubel, D., Novak, J. \& Hanesian, H. (1983). Psicología educativa. Un punto de vista cognoscitivo. México: Trías Ed.

Berko, J. (1958). The child's learning of English morfhology [en línea]. Disponible en: http://childes.psy.cmu.edu/topics/wugs/wugs.pdf

Bowey, A. \& Patel, K. (1988). Metalinguistic ability and early reading achievement. Applied Psycholinguistics, 9, 367-383.

Bybee, J. \& Slobin, D. (1982). Rules and schemas in the development and use of the English past. Language, 58, 265-289.

Carlisle, J. (1995). Morphological awareness and early reading achievement. En L. Feldman (Ed.), Morphological aspects of language processing (pp. 189-209). Mahwah, NJ: Lawrence Erlbaum.

Carlisle, J. (2003). Morphology matters in learning to read: A commentary. Reading Psychology, 24, 291-322.

Carlisle, J. (2006). Morphogical processes that influence learning to read. En C. Stone, R. Silliman, B. Ehren \& K. Apel (Eds.), Handbook of Language \& Literacy: Development and Disorders (pp. 318-339). Nueva York: The Gilford Press.

Carlisle, J. (2007). Fostering morphological processing, vocabulary development, and reading comprehension. En R. K. Wagner, A. E. Muse. \& K. R. Tannenbaum (Eds.), Vocabulary acquisition: Implications for reading comprehension (pp. 78-103). Nueva York, US: Guilford Press.

Carretero, M. (2001). Metacognición y educación. Buenos Aires: Aique. 
Casalis, S. \& Louis-Alexandre, M. (2000). Morphological analysis, phonological analysis and learning to read French: A longitudinal study. Reading and Writing: An Interdisciplinary Journal, 12, 303-335.

Cuetos, F. \& Valle, F. (1988). Modelos de lectura y dislexias. Fundación infancia y aprendizaje, 44, 3-20.

Deacon, H. \& Kirby, R. (2004). Morphological awareness: Just 'more phonological' The roles of morphological and phonological awareness in reading development. Applied Psycholinguistics, 25, 223-238.

Díaz, J. (2006). Habilidades metalingüisticas en niños alfabetizados de bajo nivel socioeconómico [en línea]. Disponible en: http://www.umb.edu.co/PDF/umbral_8.pdf

Duncan, L., Casalis, S. \& Colé, P. (2008). Early metalinguistic awareness of derivational morphology: Observations from a comparison of English and French. Applied Psycholinguistics, 30, 405-440.

Escudero, I. (2010). Las inferencias en la comprensión lectora: Una ventana hacia los procesos cognitivos en segundas lenguas [en línea]. Disponible en: http://www.nebrija.com/revista-linguistica/revista-linguisticanebrija $7 /$ index.html

Florez, R., Torrado, M., Arévalo, I., Mesa, C., Mondragón, S. \& Pérez, C. (2005). Habilidades metalingüisticas, operaciones metacognitivas y su relación con los niveles de competencia en lectura y escritura: un estudio exploratorio [en linea]. Disponible en: http: / / www.redalyc.org/articulo.oa?id=21901801

García, J. \& González, D. (2008). Batería psicopedagógica Evalúa-O. Madrid: EOS.

Gobierno de Chile, Ministerio de Educación. (2013). Programa de Estudio, Cuarto año Básico, Lenguaje y Comunicación, pag.38 [en línea]. Disponible en: http://www.curriculumenlineamineduc.cl/605/articles-18963_programa.pdf

Gobierno de Chile, Ministerio de Educación. (2012). Currículum nacional. Bases curriculares, indicadores de evaluación, programas de estudio y decretos [en línea]. Disponible en: http://www.curriculumenlineamineduc.cl/605/w3-article30013.html

González, A. (2004). Estrategias de comprensión lectora. Madrid: Síntesis.

González, L., Rodríguez, C. \& Gázquez, J. (2011). Aproximación al concepto de conciencia morfológica: Concepto, desarrollo y relación con la lectura y la escritura. Revista de Formación del Profesorado e Investigación Educativa, 24, 135-146. 
González, M., Barba, M. \& González, A. (2010). La comprensión lectora en educación secundaria. Revista Iberoamericana de Educación, 53(6), 2-11.

Hernández, R., Fernández, C. \& Baptista, M. (2010). Metodología de la investigación. Quinta Edición. México: McGraw-Hill.

Kamhi, A. \& Catts, H. (2005). Language and reading disabilities (2nd ed.). Boston: Pearson Education, Inc.

Lawshe, C. (1975). A quantitative approach to content validity. Personnel Psychology, 28, 563-575.

Mahony, D., Singson, M. \& Mann, V. (2000). Reading ability and sensitivity to morphological relations. Reading and Writing: An Interdiciplinary Journal, 12(3-4), 191-218.

Nagy, W., Anderson, R., Schommer, M., Scott, J. \& Stallman, A. (1989). Morphological families in the internal lexicon. Reading Research Quarterly, 24, 263-282.

Nagy, W. \& Scott, J. (2000). Vocabulary processes. En M. Kamil, P. Mosenthal, P. D. Pearson \& R. Barr (Eds.), Handbook of Reading Research, Volume III (pp. 269284). Mahwah, NJ: Lorence Erlbaum.

Nicasio, J. \& González, L. (2006). Diferencias en la conciencia morfológica, la escritura y el lenguaje en función del desarrollo y el nivel educativo del niño. Psicothema, 18(2), 171-179.

OCDE. (2000). Literacy In The Information Age: Final report of the international adult literacy survey. [en linea]. Disponible en: http://www.oecd.org/edu/skills-beyondschool/41529765.pdf

Owens, R. (2003). Desarrollo del lenguaje. Quinta edición. Buenos Aires: Pearson Educación S.A.

Paris, S., Cross, D. \& Lipson, M. (1984). Informed strategies for learning: A program to improve children's reading awareness and comprehension. Journal of Educational Psychology, 76(6), 1239-1252.

Pérez, M. (2005). Evaluación de la comprensión lectora: Dificultades y limitaciones. Revista de Educación, Número extraordinario, 121-138.

Puyuelo, M. (2007). BLOC-R: Evaluación del lenguaje. España: Elsevier Masson.

Sadowsky, S. \& Martínez, R. (2004). Lista de frecuencias de palabras del castellano de Chile (Lifach). Versión 1.0/1.1. Base de datos electrónica [en línea]. Disponible en: http://sadowsky.cl/lifcach.html 
Schunk, D. (2005). Self-regulated learning: The educational legacy of Paul R. Pintrich. Educational Psychologist, 40, 85-94.

Stone, C., Silliman, R., Ehren, B. \& Apel, K. (2004). Handbook of language \& literacy: Development and disorders. Nueva York: The Gilford Press.

Vallés, A. (2005). Comprensión lectora y procesos psicológicos. Revista de Psicología, 11, $49-61$.

Valtín, R. (1984). The development of metalinguistic abilities in children learning to read and write. En J. Downing \& R. Valtin (Eds.), Language awareness and learning to read (pp. 207-226). Nueva York. Springer Verlag.

\section{*AGRADECIMIENTOS}

Agradecemos a la Mg. Tilma Cornejo, por su guía y colaboración en la realización de la investigación enmarcada en la tesis para optar al grado académico de Magister en Didáctica de la Lengua Materna. 